\title{
Entgrenzung von Arbeit und Familie - mehr als Prekarisierung
}

\section{Michaela Schier Karin Jurczyk Peggy Szymenderski}

Entgrenzte Erwerbsarbeit ist heute keine exotische Ausnahme mehr. Sie trifft in einer oft schwierigen Gemengelage auf die gleichzeitig stattfindende Entgrenzung von Familie sowie von Geschlechterverhältnissen. Eine qualitative Studie über den Alltag erwerbstätiger Eltern in der Filmwirtschaft sowie dem Einzelhandel zeigt, dass die Herstellung einer gemeinsamen Lebensführung schwierig werden kann, wenn sich die raum-zeitlichen Bedingungen des Arbeitens und Lebens flexibilisieren. Inwiefern diese Entgrenzungsprozesse prekarisierend wirken, hängt jedoch vom gesamten Lebenszusammenhang und von den Umgangspraktiken der Betroffenen ab.

\section{Entgrenzung - Prekari- sierung: Deutungen sozialen Wandels}

In den Debatten um die „Krise der Arbeitsgesellschaft" haben sich in den Sozialwissenschaften seit Ende der 1990er Jahre mit „Entgrenzung“ und „Prekarisierung“ zwei Begriffe zur Beschreibung des gesellschaftlichen Wandels etabliert, die eine gewisse Verwandtschaft, jedoch auch gewichtige Unterschiede aufweisen. Beide Begriffe werden erstens als zeitdiagnostische Kategorien genutzt, um Veränderungen im über Jahrzehnte stabilen strukturellen Gefüge von modernen Gesellschaften zu thematisieren (Castel/ Dörre 2009; Gottschall/Voß 2003). In ihrem Fokus steht zweitens die Erosion des „Normalarbeitsverhältnisses“, wie es im Zusammenhang mit der Industrialisierung und Entwicklung des Wohlfahrtsstaates entstanden ist. Die Vorstellung einer zunehmenden Brüchigkeit, Ausdünnung und zum Teil auch Auflösung bis dahin für sicher gehaltener struktureller $\mathrm{Ab}$ - und Begrenzungen von Sphären der Gesellschaft und des persönlichen Lebens sowie deren Folgen für die Lebensverhältnisse bzw. Lebensführung bilden somit den Kern beider Ansätze. ${ }^{1}$

Im Prekarisierungsdiskurs wird mit Blick auf die strukturellen Merkmale von Erwerbsverhältnissen jedoch fast ausnahmslos die zunehmende strukturelle Unsicherheit im Hinblick auf die Existenzsicherung, die soziale Absicherung sowie die gesellschaftliche Integration und damit die Gefahr hervorgehoben, von etablierten
Formen der Partizipation und Chancen zur Lebensplanung ausgeschlossen zu werden (Castel/Dörre 2009). Im Mittelpunkt stehen Prozesse der Entsicherung von bislang gültigen Regulierungen der Beschäftigungs- und Arbeitsverhältnisse. Meist wird angenommen, dass mit der Prekarisierung ein Verlust von Handlungsspielräumen und Autonomie verbunden ist. Erst wenige Arbeiten fokussieren auf individuelle Verarbeitungsformen von unsicheren Beschäftigungsformen und weisen auf Unterschiede von prekären Erwerbslagen hin, auch in Verbindung mit unterschiedlichen Haushaltskonstellationen und Geschlechterarrangements (Manske 2010; Bartelheimer in diesem Heft).

Im Entgrenzungsdiskurs wird dagegen stärker auf widersprüchliche sowie auch positive Wirkungen hingewiesen, die mit den Deregulierungs- und Flexibilisierungsprozessen einhergehen. Ausgehend von der Annahme, dass durch die Entstrukturierung neue Handlungsspielräume entstehen, andererseits jedoch zugleich die Anforderungen an die Konstruktion und Stabilisierung von handlungsleitenden Strukturen steigen (Giddens 1988), lenkt die Entgrenzungsdebatte den Blick stärker auf neue Handlungsanforderungen, wie z.B. die Notwendigkeit des aktiven (Neu-) Strukturierens. Der Entgrenzungsbegriff bezieht sich nicht nur auf Entdifferenzierungsprozesse im Erwerbsbereich, sondern auch auf die Erosion von Normalitäten in anderen Bereichen, wie etwa der Familie, und ermöglicht dadurch, das Wechselspiel der aktuell gleichzeitig ablaufenden Entstrukturierungsprozesse zueinander zu beobachten (Jurczyk et al. 2009).

Dies ist von Bedeutung, weil nicht nur die Veränderungen im Erwerbsbereich, sondern auch die im Bereich von Familie und den Geschlechterverhältnissen in ihrer jeweils spezifischen Gemengelage einen neuen Bezugsrahmen für individuelle und familiale Lebensführungen schaffen und neue Alltagspraktiken sowohl notwendig als auch möglich machen. Im Folgenden beziehen wir die Diskussionsstränge zur Entgrenzung und Prekarisierung aufeinander, indem wir fragen, inwiefern sich die strukturellen Umgestaltungen sowie die normativen Verschiebungen im Erwerbsbereich („Entgrenzungen“) prekarisierend auf individuelle und familiale Lebensführungen auswirken, oder ob sich durch sie auch neue Gestaltungsmöglichkeiten für das Privatleben und für Geschlechterarrangements ergeben.

\footnotetext{
Beide Konzepte wurden für ihre Genderblindheit kritisiert. Inzwischen gibt es jedoch Analysen, die die Geschlechterperspektive integrieren (Schier/ Szymenderski 2009).
}

Michaela Schier, Dr., leitet die SchumpeterNachwuchsgruppe "Multilokalität von Familie" am Deutschen Jugendinstitut (DJI), München. Arbeitsschwerpunkte: Multilokalität, familiale Lebensführung, Gender. e-mail: schier@dji.de Karin Jurczyk, Dr., leitet die Abteilung Fami lie und Familienpolitik am DJI. Arbeitsschwerpunkte: Familie, Lebensführung, Gender. e-mail: jurczyk@dji.de Peggy Szymenderski, Dipl. Soz., Mitarbeiterin im Frauenbildungshaus Dresden e.V., Promotion über "Gefüh/sarbeit im Polizeidienst". Arbeitsschwerpunkte: Lebensführung, Gefüh/sarbeit, Polizei. e-mail: peggy.szymenderski@gmx.de 
Vor dem Hintergrund eines empirischen Forschungsprojektes (Jurczyk et al. 2009) wollen wir die Schattenseiten der Entgrenzung ausleuchten und so Bezüge zur Prekarisierungsdebatte herstellen. Gleichzeitig soll deutlich werden, dass Letztere eine negative Schlagseite hat und dadurch wichtige Dimensionen des sozialen Wandels verfehlt.

$\mathrm{Zu}$ Beginn des Beitrags wird die konzeptionelle Figur der doppelten Entgrenzung skizziert (Abschnitt 2). Anhand konkreter empirischer Ergebnisse wird im Hauptteil des Beitrags aufgezeigt, welche Herausforderungen sich durch die doppelte Entgrenzung den Individuen und Familien in ihrem Alltag stellen (Abschnitt 3). Abschließend werden die negativen und positiven Potenziale der beschriebenen Entgrenzungen resümiert und daraus folgende Differenzierungen für die aktuelle Prekarisierungsdebatte herausgearbeitet (Abschnitt 4).

\section{Doppelte Entgrenzung: Erosion von Normalitäten}

Im Vergleich zu den 1950/60er Jahren haben in den Sphären von Arbeit und Leben inzwischen grundlegende Veränderungen stattgefunden: Seinerzeit fand Erwerbsarbeit typischerweise außerhalb des privaten Raums in dafür reservierten Räumen statt, es dominierte das sogenannte Normalarbeitsverhältnis als sozialrechtlich abgesicherte Vollzeiterwerbsarbeit mit geregelten Arbeitszeiten ebenso wie die „Normalfamilie“, d.h. üblicherweise verheiratete Eltern lebten mit ihren leiblichen Kindern in einem gemeinsamen Haushalt zusammen. Ferner gab es eine klare geschlechtsspezifische Arbeitsteilung, der zufolge Frauen zuständig für Haushalt und Kinder, Männer als Ernährer der Familie galten.

Seit den späten 1960er Jahren ist in Deutschland ein simultan verlaufender und sich gegenseitig beeinflussender mehrfacher struktureller Wandel von Erwerbsarbeit sowie der privaten Lebensverhältnisse und Geschlechterarrangements zu erkennen. ${ }^{2}$ Das Normalarbeitsverhältnis hat in den letzten Jahrzehnten zugunsten "atypischer" Beschäftigungsformen, wie Teilzeitarbeit, befristete oder geringfügige Beschäftigung, an Bedeutung verloren. Damit einher ging eine massive zeitliche
Entstandardisierung und Flexibilisierung in Lage, Dauer und Verteilung von Arbeitszeiten. Erwerbsarbeit löst sich tendenziell von der Bindung an bestimmte Orte des Arbeitens. Erwerbsbiografische Unsicherheiten haben zugenommen. Die bisherige, vor allem für Männer gültige Normalbiografie als eine klare Abfolge von Phasen der Ausbildung, stabiler Berufstätigkeit und Ruhestand erodiert.

Die Veränderungen in den Familien zeigen sich an einer größeren Vielfalt an Haushalts- und Familienformen. Immer mehr Erwachsene und Kinder erleben im Verlauf ihres Lebens mehrfache Wechsel zwischen verschiedenen familialen Settings. Im Anschluss an eine Trennung der Eltern spielt sich das Familienleben in unterschiedlicher Intensität in verschiedenen Haushalten ab. Familien sind heute zunehmend haushaltsübergreifende, multilokale Netzwerke. Die raum-zeitliche Eingebundenheit der Familienmitglieder außerhalb der Familie wird komplexer. Männer verstehen sich nicht mehr nur als Familienernährer. Insbesondere junge Väter beschränken ihre Erwerbsarbeit in bestimmten Segmenten und Phasen, beispielsweise durch Teilhabe an der Elternzeit.

Gesellschaftliche Grenzziehungen (z.B. zwischen Arbeitszeit und Freizeit, männlichen und weiblichen Zuständigkeiten), Normen (z.B. bezüglich Arbeitsbedingungen, Einkommen), Leitbilder (z.B. bezüglich Vaterschaft, Müttererwerbstätigkeit) und Wertvorstellungen, die sich im Verlauf der Industrialisierung für Erwerbsarbeit, Familie sowie ihre jeweilige geschlechtsspezifische Strukturierung etablierten, sind damit brüchig geworden oder haben sich sogar grundlegend geändert. Zusammenfassend beschreibt diese Prozesse der sozialwissenschaftliche Begriff der „Entgrenzung “ (Gottschall/Voß 2003). Da die Veränderungsprozesse nicht nur im Erwerbsbereich, sondern auch in der Familie stattfinden, sprechen wir von einer doppelten Entgrenzung. Die fordistische Trennung von „Arbeit und Leben“, die konstitutiv mit einer traditionellen geschlechtsspezifischen Arbeits- und Rollenteilung verbunden war, gerät durch Strukturwandlungen in beiden Sphären unter Druck. Die Erosion des bisherigen arbeitsteiligen Verhältnisses von Familie und Erwerb erfordert von den Beschäftigten ein permanentes Ausbalancieren zwischen beiden Bereichen im Alltag und im Lebensverlauf.

\section{Familiale Lebensführung unter Entgrenzungsbedin- gungen}

In Familien verknüpfen sich je nach $\mathrm{Fa}$ milienkonstellation, Geschlechterarrangements sowie Erwerbskonstellationen Prozesse der Entgrenzung von Familie mit der Entgrenzung von Erwerbsarbeit $\mathrm{zu}$ eigenen, komplexen familialen „Gemengelagen" der doppelten Entgrenzung. Diese werden wiederum im Rahmen der familialen Lebensführung auf spezifische Art und Weise verarbeitet. Familien sind hierbei gefordert, Praktiken zu entwickeln, mit denen sie den neuen Anforderungen begegnen können und die zu neuen $\mathrm{Ba}$ lancen führen.

Eine Annäherung an unsere Fragestellung, inwiefern die Entgrenzungen der Erwerbsarbeit prekarisierend auf Lebensführungen wirken, oder ob sich vielmehr auch neue Gestaltungsmöglichkeiten für private Lebensverhältnisse und Geschlechterarrangements ergeben, ist deshalb nur unter Berücksichtigung von Kontexten - Branchen, Berufe und Regionen sowie Familienkonstellationen - möglich. Wir konzentrieren uns auf zwei Berufsfelder, die wir im Forschungsprojekt „Entgrenzte Arbeit - entgrenzte Familie“ näher untersucht haben. ${ }^{3}$ Im Rahmen einer Kooperation zwischen dem Deutschen Jugendinstitut und der Technischen Universität Chemnitz wurden in Leipzig und München mit 30 Vätern und 46 Müttern mit Kindern im Alter von 0 - 16 Jahren Interviews zu ihrem Alltag unter Entgrenzungsbedingungen geführt. Die Eltern waren sowohl auf höheren als auch niedrigen Erwerbspositionen im Einzelhandel sowie dem Bereich der Filmproduktion tätig.

\subsection{PREKARISIERUNG DURCH ENTGRENZTE ERWERBSARBEIT?}

Auf den ersten Blick tragen die Erwerbsbedingungen beider untersuchten Gruppen sowohl hinsichtlich der Arbeits- als auch

2 Zu unterschiedlichen Entwicklungslinien, Strukturaspekten und zugrunde liegenden Normalitätsfolien der Entgrenzung in Ost- und Westdeutschland siehe Jurczyk et al. (2009).

3 Diese Studie (Jurczyk et al. 2009) wurde von der Hans-Böckler-Stiftung und dem Deutschen Jugendinstitut gefördert. 
Beschäftigungsverhältnisse etliche Kennzeichen, die in der Prekarisierungsdebatte üblicherweise als, prekarisierend'beschrieben werden (z.B. Brinkmann et al. 2006).

\section{ERWERBS- UND EINKOMMENS- UNSICHERHEIT ALS NORMALITÄT}

In der Filmbranche stieg seit Mitte der 1980er Jahre mit der Privatisierung und Entbetrieblichung der Branche vor allem die Zahl der auf Produktionsdauer befristet Angestellten sowie die der „freien“ Beschäftigten bei einer gleichzeitigen Abnahme der regulären Vollzeitbeschäftigung (Marrs 2007). Insbesondere für die kurzfristig Beschäftigten, zu denen die meisten unserer Befragten zählen, gehören aufgrund der Saisonalität des Filmgeschäfts und fehlender direkter Projektanschlüsse periodische Phasen der Arbeitslosigkeit zu ihrer „normalen“ Berufsbiografie. Dies gilt für Männer wie auch für Frauen. Durch diese Erwerbspausen sowie eine untertarifliche Bezahlung besteht für viele das Risiko zu verarmen (Satzer 2007). ${ }^{4}$ Die Verkürzung der Rahmenfrist im Zuge der Hartz-Reformen im Jahr 2006 schloss zudem Filmschaffende, die u.a. aufgrund ihrer familialen Verpflichtungen nur wenige Projekte pro Jahr annehmen können, zum Zeitpunkt unserer Studie aus dem Bezug von Arbeitslosengeld I aus. ${ }^{5}$ Das Verarmungsrisiko von Frauen ist hierbei höher, da sie eher in schlechter bezahlten Positionen arbeiten und die meisten der familialen Pflichten übernehmen.

Auch im Einzelhandel stieg in den letzten zehn Jahren die Zahl der Teilzeit- und geringfügig Beschäftigten zuungunsten der regulär in Vollzeit Tätigen. Die Beschäftigungsstrukturen innerhalb der Branche unterscheiden sich erheblich nach Geschlecht. Während fast drei Viertel der Männer in Vollzeit arbeiten, dominiert bei Frauen mit gut $60 \%$ die Teilzeitarbeit bzw. geringfügige Beschäftigung (BMWA 2005, S. 198). Die Zahl der Männer in Teilzeit stieg jedoch in nur vier Jahren bis 2006 um rund $18 \%$ (Statistisches Bundesamt 2003, 2007). Der harsche Preiswettbewerb, dem Unternehmen mit der Senkung ihrer Personalkosten begegnen, erzeugt bei den Beschäftigten fühlbaren Druck. Unsere Befragten weisen deutlich auf eine latente Bedrohung durch Entlassungen hin, wenn auch in der untersuchten ostdeutschen Region mehr als in der westdeutschen. Die insgesamt schlechtere Arbeitsmarktlage im Osten Deutschlands sowie frühere Erfahrungen mit Arbeitslosigkeit scheinen individuelle Ängste zu verstärken. Noch ausgeprägter als Filmschaffende sind Beschäftigte im Einzelhandel von niedrigen Einkommen und deshalb in ihrer Lebensführung von finanziellen Engpässen betroffen (Statistisches Bundesamt 2009).

Die Erwerbsbedingungen sind damit sowohl im Einzelhandel als auch in der Filmbranche als durchaus materiell prekär sowie in Bezug auf die soziale Absicherung und Erwerbskontinuität als unsicher zu bezeichnen. Doch nur bei einigen der Befragten äußern sich die finanziellen und biografischen Unsicherheiten als Existenzängste. Insbesondere Filmschaffende betonen ebenso Vorteile ihrer befristeten Beschäftigung sowie der erwerbslosen Phasen zwischen Projekten für sich selbst und die Familie.

\section{RISIKEN FÜR GESUNDHEIT UND SOZIALE EINBINDUNG}

Neben den erwerbsbiografischen Risiken fordern zeitliche und räumliche Entgrenzungen eine hohe Verfügbarkeit und Flexibilität und führen zu Planungs- und Gestaltungsunsicherheiten der Lebensführungen der Beschäftigten. In beiden Branchen werden zudem regelmäßig Arbeits- und Gesundheitsschutzstandards unterschritten und die Beschäftigten damit hohen körperlichen und psychischen Belastungen ausgesetzt.

Die Arbeitszeiten der Filmschaffenden sind auf allen Hierarchieebenen extensiv. Während der Dreharbeitsphasen, die regelmäßig als „Leben im Ausnahmezustand“ geschildert werden, gelten tägliche Arbeitszeiten von zehn Stunden als absolutes Minimum. Die durchschnittliche effektive Wochenarbeitszeit liegt bei 50 Wochenstunden, Überstunden und Mehrarbeit gehören zum Arbeitsalltag (Satzer 2007). Dabei ist Arbeit zu „unsozialen“ Zeiten weit verbreitet - gedreht wird auch nachts, an Wochenenden und Feiertagen. Hinzu kommen Belastungen durch außergewöhnliche körperliche und mentale Anstrengungen, die häufig suboptimale Unterbringung am Arbeitsort sowie die ausgeprägte Fremdsteuerung des Tagesablaufs durch die tägliche Disposition. Räumliche Mobilität ist für Filmschaffende ein Muss, da sie keinen festen Arbeitsort haben sowie Wohn- und Drehort nur sehr selten zusammenfallen. Reisen im In- und Ausland, ein Leben in
Hotels und Pensionen sowie wochen- bis monatelange Abwesenheiten von der Familie gehören zum Alltag. Die Aufträge für Produktionen werden hierbei oft sehr kurzfristig vergeben, was den Beschäftigten eine enorme Flexibilität abverlangt.

Im Einzelhandel sind durch die Liberalisierung der Ladenöffnungszeiten inzwischen Betriebszeiten von über 60 Wochenstunden, sozial und familial ungünstig gelegene Arbeitszeiten am Abend, nachts und am Samstag verbreitet. Der Trend zu flexiblen Arbeitszeiten und hochflexiblen Schichtsystemen hat sich erheblich verstärkt. Davon sind die meist weiblichen Teilzeitkräfte besonders betroffen. Auch hier ist die Arbeitszeitplanung oft extrem kurzfristig, was die Planung des außerbetrieblichen Lebens erschwert. Für Vollzeit- und Führungskräfte sind Arbeitszeiten zwischen 55 und 70 Stunden sowie die Erwartung, Rund-um-die-Uhr für den Betrieb verfügbar zu sein, typisch (Groß et al. 2007). Vor dem Hintergrund der ungleichen Verteilung von Männern und Frauen auf unterschiedliche Erwerbsformen und Statuspositionen im Einzelhandel trifft das vor allem auf Männer zu. Für Führungskräfte spielen räumliche Mobilitätsanforderungen eine nicht unerhebliche Rolle. Ein beruflicher Aufstieg geht häufig mit einer höheren Reisetätigkeit oder dem Wechsel des Arbeitsortes einher. Ihre Arbeit hat sich überdies aufgrund von Rationalisierungsmaßnahmen drastisch verdichtet (Ver.di 2006). Verkaufstätigkeiten sind heute zudem mit hohen Anforderungen an die Selbstorganisation innerhalb oftmals restriktiver Rahmenbedingungen sowie an die Kommunikativität und Gefühlsarbeit verbunden (Voss-Dahm 2004). Unternehmen setzen zur Bewältigung der Flexibilitätsanforderungen außerdem auf eine hohe funktionale Flexibilität.

Bei beiden befragten Gruppen sind demnach erhebliche Belastungen durch Selbstausbeutung, ein nicht nachhaltiger Umgang mit der eigenen Arbeits- und Le-

\footnotetext{
4 Knapp die Hälfte der Beschäftigten in der Branche verfügte im Jahr 2006 über ein Bruttojahresentgelt von bis zu 30.000 Euro (Satzer 2007, S. 24).

5 Der Gesetzgeber hat 2009 für kurzfristig Beschäftigte eine verkürzte Anwartschaft auf Arbeitslosengeld I sowie eine abgestufte Anspruchsdauer eingeführt. Der BundesFilmVerband (BFV) bezweifelt jedoch die Verbesserung der sozialen Absicherung professionell Filmschaffender, vgl.: http://www.connexx-av.de.
} 
benskraft und damit Risiken im Hinblick auf die physisch-psychische Gesundheit feststellbar. Beim Verkaufspersonal sind bei Einzelnen zudem Sinnverluste, Statusunsicherheit, Anerkennungs- und Planungsdefizite sowie eine Schwächung sozialer Netze zu finden, die nach Brinkmann et al. (2006) subjektive Sichtweisen prekär Beschäftigter kennzeichnen. Bei der Gruppe der Filmschaffenden sowie einigen der Führungskräfte im Einzelhandel fallen jedoch eine hohe intrinsische Motivation zur Ausübung der Tätigkeit sowie eine verbale Normalisierung der extremen Erwerbsbedingungen auf (Betzelt 2008).

\subsection{UMGANG MIT PREKARI- SIERUNGSRISIKEN}

Ohne Zweifel bestehen bei beiden Befragtengruppen Risiken der materiellen und sozialen Verarmung, des Verlusts an Eingebundenheit in lokale Öffentlichkeiten, im Hinblick auf die eigene Gesundheit, aber auch auf die Herstellung von Familie. Zudem existieren beträchtliche Planungsund Gestaltungsunsicherheiten sowohl in der Alltagsdimension, bei der Vereinbarkeit von Sorge- und Erwerbsarbeit, als auch in der biografischen Dimension. Gezeigt hat sich jedoch ebenso, dass die vielfältigen Trends der Entgrenzung von Erwerbsarbeit erstens nicht einheitlich und per se prekarisierend wirken und zweitens den Alltag der Befragten und ihrer Familien nicht determinieren. Vielmehr entwickeln die Beschäftigten und ihre Familien zahlreiche Praktiken, um mit den Unsicherheiten, Unvereinbarkeiten und Belastungen umzugehen. Allerdings gelingt dies nicht allen in gleicher Weise. In unserer Studie konnten wir einige Variablen identifizieren, welche einzeln und in ihrer Summe die Entwicklung von Umgangspraktiken moderieren und damit den Ausschlag geben, ob Entgrenzungen für die Akteure eher chancen- oder risikoreich sind.

\section{DER UMGANG MIT UNSICHER- HEITEN}

Den Alltag mit Einkommens- und Planungsunsicherheiten zu bewältigen, ist eine zentrale Anforderung an die Beschäftigten und ihre Familien im Einzelhandel und in der Filmbranche. Ökonomische Risiken und hohe Flexibilitätsanforderungen werden dabei vielfach durch die familialen Erwerbskonstellationen und - damit eng verbunden - die Arbeitsteilungsmuster zwischen den Partnern abgefedert. Im Vergleich zu den Filmschaffenden sind bei den meisten Einzelhandelsfamilien deutlichere Hinweise darauf zu finden, dass die Erwerbseinbindung beider Elternteile ökonomisch bedingt ist. Einige filmschaffende Paare versuchen, die unsichere Beschäftigungs- und Einkommenssituation des Partners durch ein geregeltes, meist jedoch nicht existenzsicherndes Erwerbsverhältnis der Partnerin zu kompensieren. Dieser Praxis sind jedoch durch das branchenspezifisch geringe Angebot von unbefristeten Stellen externe Grenzen gesetzt. Die befragten alleinerziehenden Frauen können hingegen während ihrer beruflichen Abwesenheiten nicht auf einen Partner im Hintergrund zurückgreifen - weder um die finanzielle Existenz zu sichern, noch um familiale Aufgaben zu bewältigen. Ihre Möglichkeiten, mit den Unsicherheiten und Flexibilitätsanforderungen umzugehen, hängen verstärkt davon ab, wie sie in persönliche Netzwerke sowie das berufliche Umfeld eingebunden sind.

Private Lebensverhältnisse und Geschlechterverhältnisse können jedoch ebenso erwerbsbedingte Prekarisierungsrisiken verstärken - das lässt sich nicht nur am Beispiel der Konsequenzen von Trennungen verdeutlichen, sondern auch an der Widersprüchlichkeit von aktuellen Männlichkeitskonzepten. Trotz niedriger Einkommen, unsteter Erwerbsbiografien sowie gestiegenen - auch eigenen - Ansprüchen an die Ausübung einer aktiven Vaterschaft halten die meisten Männer in unserer Studie an einer traditionellen männlichen Selbstdefinition als Hauptverantwortlicher für die finanzielle Existenzsicherung der Familie fest. Um dieser Rolle trotz der genannten Probleme gerecht zu werden, engagieren sich Männer vielfach verstärkt im Erwerbsbereich. Sie nehmen Zweitjobs an, lehnen auch weniger gute Projekte nicht ab, akzeptieren extrem lange Arbeitszeiten und ungünstige Mobilitätsanforderungen. Sie gefährden damit nicht nur ihre eigene Gesundheit, sondern nehmen auch in Kauf, in das Leben der Familie nur marginal eingebunden zu sein. Insofern verweisen unsere Ergebnisse, wie andere Studien auch, auf den Zusammenhang von Prekarisierungswirkungen von Erwerbsformen und dem Vorhandensein von sozialen Ressourcen, der Haushaltszusammensetzung sowie den Geschlechterarrangements.
Der Blick auf die Lebensführung von Filmschaffenden verweist auf die besonderen Gestaltungsnotwendigkeiten, die aus deren spezifischen erwerbsbiografischen Risiken resultieren: Gerade der Übergang von der intensiven Einbindung in Erwerbsarbeit zur Nichterwerbsphase sowie der Alltag während der Nichterwerbsphasen zwischen Filmprojekten sind besonders sensible Momente der familialen Lebensführung. Das Ende eines Filmprojekts bedeutet in der Regel einen radikalen Bruch, der Alltag muss wieder selbst strukturiert und es muss mit viel freier Zeit umgegangen werden. Diese Anforderung gelingt nicht allen Befragten gleich gut. Hier zeigt sich, dass die Familie zur Bewältigung dieses Übergangs eine wichtige Funktion als psychische Stabilisatorin und Strukturgeberin des Alltags einnimmt. Insbesondere die Partnerinnen übernehmen für die beruflich phasenweise abwesenden Männer grundlegende sozial-integrative Funktionen. Dauert die - meist positiv konnotierte - projektfreie Zwischenphase länger als gewollt, sodass finanzielle Engpässe drohen, fällt es den Befragten zunehmend schwerer, ihre freie Zeit positiv zu nutzen (Rogge 2009). Um der zunehmenden innerlichen Anspannung und Konflikten in der Familie zu begegnen, entwickeln die Befragten Praktiken, wie das Aufbauen eines zweiten beruflichen Standbeins oder subtile Telefonakquise.

\section{DER UMGANG MIT ZEITLICH- RÄUMLICHEN ENTGRENZUNGEN}

Auf der Basis der je spezifisch raum-zeitlich entgrenzten Erwerbsbedingungen sind in unserem Sample im Vergleich zur fordistischen Familie unterschiedliche neue Raum-Zeit-Muster der Anwesenheit der Eltern in der Familie zu erkennen, von denen wir hier drei vorstellen:

- das Muster der dauerhaft knappen Kopräsenz,

- das Muster des phasischen Wechsels von knapper und ausgiebiger Kopräsenz sowie - das Muster der zerstückelten Kopräsenz.

Jedes dieser Muster erfordert spezifische praktische Gestaltungsleistungen und geht mit eigenen Problemen und Chancen der familialen Lebensführung einher.

Die beiden ersten Muster sind typisch für Familien von Vollzeit- und Führungskräften im Einzelhandel sowie für die 
Familien der meisten Filmschaffenden. Diese Eltern sind häufig zu Zeiten nicht zu Hause, in denen zahlreiche familiäre Aufgaben anfallen: in den Abendstunden sowie an den Wochenenden. Beobachtbar ist, dass hier - meist vermittelt durch eine traditionale geschlechtsbezogene Arbeitsteilung - vielfach die beruflich weniger eingespannte Partnerin versucht, die sehr knappe Familienzeit ihres Mannes auszugleichen. Jedoch speziell in Familien, in denen beide Elternteile zeitlich stark in Erwerbsarbeit eingebunden sind, aber auch bei Müttern und Vätern in hochvariabler Teilzeitarbeit im Einzelhandel, kommt es vor allem in den Abendstunden und an Samstagen zu Betreuungsengpässen. Familien stehen deshalb vor der Herausforderung, ein alternatives Betreuungsnetz aufzubauen. Wenn Großeltern verfügbar sind, wird meist auf sie zurückgegriffen. Erziehung und Betreuung älterer Kinder werden zudem vermehrt aus der Distanz über das Telefon geleistet. Familien mit dauerhaft knapper Kopräsenz können trotz dieser schwierigen Rahmenbedingungen zwar die technisch-organisatorischen Seiten (also die „Logistik“) des Alltags bewältigen, die emotionalen und gemeinschaftlichen Momente, welche die Qualität von Familienleben ausmachen, kommen jedoch häufig zu kurz. Bei Familien, in denen sich Phasen mit knapper und ausgiebiger Anwesenheit von Elternteilen periodisch abwechseln, wie das bei den meisten Filmschaffenden der Fall ist, bieten die längeren Nichterwerbsphasen insgesamt bessere Möglichkeiten für die Herstellung von Familie. Sie erlauben zumindest phasenweise ein intensives Familienleben und bieten Freiräume für die Gestaltung einer engagierten Vaterschaft.

Zum dritten Muster der zerstückelten Kopräsenz führen in Lage und Dauer stark variierende Arbeitszeiten sowie eine äußerst geringe Arbeitszeitsouveränität, die typisch für die meist weiblichen Teilzeitkräfte im Einzelhandel sind und sich am extremsten in Familien zeigen, in denen beide Elternteile in hochflexiblen Schichtsystemen tätig sind. Durch die ausgeprägte De-Rhythmisierung der Arbeitszeiten ist die Anwesenheit in der Familie nicht vorhersehbar. Zudem liegen die arbeitsfreien Zeiten dieser Eltern in der Regel versetzt zu den Freizeiten der anderen Familienmitglieder. Die hohe Variabilität der familialen Raum-Zeit-Muster zieht im Alltag der Familien einen erheblichen Koordinations-,
Synchronisations- und Planungsaufwand nach sich. Auffällig oft beobachteten wir bei Familien dieses Musters ein Arbeiten in Gegenschichten, um Lücken bei der Kinderbetreuung zu vermeiden (Boushey 2006). Väter wie Mütter bemerken einerseits positiv, sich auf diese Weise ganz auf die Bedürfnisse ihrer Kinder einstellen zu können, und Männer sind so stärker in die Familienarbeit und Kinderbetreuung eingebunden. Andererseits bestehen sowohl für die Familie als Gemeinschaft als auch für Paarbeziehungen Gefahren: Es fehlt an gemeinsamer Familienzeit über die dyadischen Beziehungen hinaus sowie an Paarzeit (Lesnard 2008).

Zudem resultieren aus der multilokalen Lebensführung vieler Familien besondere Herausforderungen für die Alltagsgestaltung. So forcieren die zum Teil sehr langen Abwesenheiten beruflich mobiler Eltern Praktiken, die die emotionale Verbundenheit aus der Ferne aufrechterhalten. Regelmäßige Familientelefonkonferenzen, das Nachreisen der Familie an den Arbeitsort eines Familienmitglieds oder mobiles Familienwohnen sind Beispiele für den Umgang mit beruflicher Mobilität (Schier 2010). Telefongespräche können Face-toFace-Kontakte jedoch nur sehr bedingt ersetzen. Prekär werden das Moment der Verlässlichkeit sowie die Möglichkeiten für Beziehung stiftende beiläufige Interaktionen der Familienmitglieder, die für die erlebte Qualität von Familienzeit von großer Bedeutung sind (Kremer-Sadlik/ Paugh 2007).

Ebenso ist jedoch beobachtbar, dass erwerbstätige Eltern versuchen, die zeiträumliche Flexibilität und Verfügbarkeit ihrer Arbeitskraft bewusst einzuschränken und so überbordenden Ansprüchen aus dem Erwerbsbereich Grenzen zu setzen. Dabei zeigt sich, dass weder die Flexibilisierung von Arbeitszeiten noch ein gewisser Grad von Ortsunabhängigkeit von Arbeit an sich problematisch für die Verknüpfung von Familien- und Erwerbsleben sind. Sie kommen Eltern durchaus in gewisser Weise entgegen, eröffnen ihnen neue Möglichkeiten für die Gestaltung des Alltags (Jurczyk 2007). Problematisch sind hochflexible Arbeitszeiten und räumliche Mobilität dann, wenn die Entscheidungsbefugnisse einseitig beim Arbeitgeber liegen sowie die Arbeitszeit- und Reiseplanungen wenig verlässlich und kurzfristig sind.

\section{DER UMGANG MIT ERSCHÖPFUNG}

Sowohl die im Einzelhandel tätigen als auch die filmschaffenden Eltern sind durch extensive Arbeitszeiten, Arbeitsverdichtung, die Zerstückelung der Arbeitszeiten bei Teilzeitarbeit sowie erwerbsbiografische und finanzielle Unsicherheiten - unterschiedlich je nach Position und Tätigkeitsfeld - körperlichen und psychischen Belastungen ausgesetzt, die auch Folgen für die Familie als Gemeinschaft haben können.

Ein angespanntes Zeitbudget wirkt sich negativ auf die Interaktionen im Familienzusammenhang aus, hochflexible Schicht- und Nachtarbeit bergen die Gefahr, soziale Kontakte nicht pflegen zu können und innerfamiliale Konflikte zu fördern. Systematisch betrachtet führen die Entgrenzungen zu Zeit-, Energie- und Aufmerksamkeitskonkurrenzen, die unter bestimmten Bedingungen eine Beteiligung am Familienleben erschweren. Eltern sind häufig so erschöpft, dass sie weder zum eher pragmatischen Vereinbarkeitsmanagement, noch weniger aber zur Konstruktion familialer Gemeinschaft beitragen können. Sorgeleistungen sowie die „Herstellung von Familie“ erfolgen oft an der Grenze des Machbaren.

Im Vergleich zu den Befragten aus der Filmbranche sind die im Einzelhandel tätigen Eltern wesentlich stärker gefordert, die sensiblen, störungsanfälligen Übergänge zwischen dem Erwerbs- und dem familialen Bereich täglich zu gestalten. Es kommt dabei darauf an, schnell von den Anforderungen des Erwerbs- auf den Familienbereich „umzuschalten“, trotz der eigenen Erschöpfung gelassen im Umgang mit den Kindern sowie ansprechbar für partnerschaftliche Bedürfnisse zu bleiben. Während einige Befragte Praktiken entwickeln, um negativen emotionalen Übertragungsprozessen zu begegnen sowie Gemeinschaft fördernde Aktivitäten und Fürsorgearbeit trotz der eigenen Erschöpfung zu leisten, schaffen das andere nicht oder nur unzureichend. Die Praktiken setzen in ganz unterschiedlichen Bereichen an und reichen von Versuchen, in weniger belastende Beschäftigungsfelder zu wechseln, über die Reduzierung der eigenen raum-zeitlichen Verfügbarkeit bis hin zur bewussten Schaffung von Erholungszeiträumen.

Die Notwendigkeit, Fürsorgearbeit trotz Erschöpfung zu leisten, trifft aufgrund der Zuschreibung der doppelten Verantwortung in Familie und Arbeitswelt 
Frauen stärker als Männer. Aber auch für Männer hat sich der psychische und handlungspraktische Druck erhöht, Familie und Erwerb gut zu verknüpfen. Die Bereitschaft von Frauen, die Doppelbelastung quasi automatisch alleine zu tragen, sinkt aufgrund ihrer eigenen Erwerbstätigkeit zunehmend (vgl. Klenner et al. in diesem Heft).

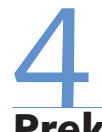

\section{Prekarisierung - abhängig von der gesamten Lebens- situation}

Entgrenzungen haben - wie beschrieben - ambivalente und nicht nur negative Wirkungen auf den gesamten Lebenszusammenhang und die Lebensführungen. Insofern leuchtet das Konzept der Entgrenzung mehr Facetten des aktuellen sozialen Wandels aus. Gleichwohl gibt es Schnittmengen zum Prekarisierungsdiskurs: Es finden sich auch in unserer Studie Hinweise auf eine Prekarisierung von Lebenszusammenhängen, die deutlich über die ökonomische Dimension hinausgehen und vor allem durch Belastungen, Destabilisierung und Verunsicherungen von Individuen, von familialen Beziehungen und sozialer Einbindung gekennzeichnet sind. Dies gilt selbst dann, wenn bestimmte Entgrenzungen individuell positiv besetzt sind und aktiv herbeigeführt wurden. Entgrenzungen können also prekär sein, müssen dies aber nicht dies hängt im Wesentlichen von Verarbeitungsformen und dem Zusammenspiel mit weiteren Ressourcen ab. Zum Verständnis von Entgrenzung und von Prekarisierung reicht demnach der Blick auf das einzelne Individuum nicht aus, sondern der Haushalts- bzw. Lebensformkontext muss mit einbezogen werden.

Für den Zusammenhang von Entgrenzungs- und Prekarisierungsdebatte ergeben sich deshalb folgende Schlussfolgerungen:

(1) Es scheint unabweisbar, dass die möglichen negativen Folgen entgrenzter Erwerbsbedingungen sich auf den gesamten individuellen und familialen Lebenszusammenhang auswirken. Dabei tragen nicht nur arbeitsvertragliche Aushöhlungen zur Entstehung von pre- kären Lebenssituationen bei, sondern auch Aspekte innerhalb der Arbeitsverhältnisse - wie etwa hoher Arbeitsdruck, Überstunden, zeitliche Flexibilisierung, Ausdehnung der Arbeitszeiten auf den Abend und das Wochenende. Zwar sind im Haushaltskontext gesehen nicht alle prekär Beschäftigten arm, allemal dann nicht, wenn sie durch Partner abgesichert sind, doch kann genau diese Abhängigkeit bei Trennung oder auch langfristig zu Armut im Alter führen.

(2) Die Prekarisierung von Erwerbsarbeit gewinnt ihre negative Bedeutung manchmal erst durch das Zusammenspiel mit anderen Belastungen im Lebenszusammenhang. Dabei können Faktoren der Destabilisierung, die aus dem Familienzusammenhang hinzutreten oder die die Erwerbssituation sogar überlagern, die erwerbsbedingten Belastungen verschärfen. Partnerschaften und Haushalte können in diesem Zusammenhang eine Ressource, z.B. zur Abfederung eines geringen Einkommens, gleichzeitig aber auch eine Belastung sein, z.B. bei Familienkonflikten (vgl. Amacker in diesem Heft).

(3) Manche, objektiv als prekarisierend zu bewertenden Merkmale von Erwerbsarbeit werden subjektiv nicht als solche wahrgenommen. So kann subjektiv Teilzeitarbeit oder eine Alleinselbstständigkeit im Vergleich zu einer Vollzeittätigkeit als die bessere Wahl für die Balance von Arbeit und Leben erscheinen oder sogar eine Erwerbsbeteiligung erst ermöglichen. Da es hier um Deutungen und Einstellungen geht, können Selbsttäuschungen durchaus eingeschlossen sein. Dieses individuelle Abwägen von Vor- und Nachteilen geschieht vor dem Hintergrund von Geschlechter- und Familienkonzepten, die eine wichtige moderierende Rolle bei der Deutung von Prekarisierungsrisiken spielen. Prekarisierende Elemente werden möglicherweise in Kauf genommen in Relation zu einer anderen, (noch) weniger passenden Gesamtfiguration von Leben und Arbeiten.

(4) Entgrenzte Erwerbsbedingungen determinieren auch in ihrer prekären Form keineswegs den Alltag der Beschäftigten und ihrer Familien, sondern diese entwickeln Umgangspraktiken mit den Prekarisierungsrisiken. Die zeitliche Flexibilisierung, Befristung und räumliche Mobilität nutzen sie teilweise bewusst für eigene Interessen sowie für die Verknüpfung von Familie und Beruf. Teils treiben sie Entgrenzungen selbst voran, indem sie Einfluss auf ihre Arbeitsbedingungen nehmen und so ihre familiale Lebensführung eigensinnig zu gestalten versuchen.

(5) Und schließlich scheint die Akzeptanz prekärer Erwerbskonstellationen insbesondere für Frauen ein, wenngleich riskanter, Ausweg aus traditionalen Abhängigkeiten vom Ehemann zu sein. Denn die Entgrenzung starrer Arbeits- und Lebenswelten, die an traditionalen Geschlechterverhältnissen und am männlichen Normalarbeitsverhältnis orientiert waren, birgt auch Chancen: für mehr Erwerbsbeteiligung der Mütter, weniger hierarchische Verhältnisse zwischen den Geschlechtern und mehr Zeit der Kinder mit ihren Vätern.

Die Entgrenzung von Erwerbsarbeit entfaltet ihre prekarisierende oder aber unterstützende Wirkung erst, wenn sie im Kontext des gesamten Lebenszusammenhangs gesehen wird. Die konzeptionelle Dimension der „Vereinbarkeitssicherheit" als „Möglichkeit, Erwerbsarbeit und Sorgearbeit in der persönlichen Lebensführung kombinieren zu können" (Bartelheimer in diesem Heft) erfährt so eine durchaus individualistische Wendung - allerdings vor dem Hintergrund, dass Rahmenbedingungen für die Balance von Arbeit und Leben gesellschaftlich vorstrukturiert sind.

Trotz positiver Potenziale von Entgrenzungen gilt unweigerlich, dass diese den Alltag von Familien komplexer machen. In den vielfältigen Anzeichen von Erschöpfung erwerbstätiger Eltern, die dadurch entsteht, dass Erwerbsarbeit, sozialstaatliche Regelungen, Familienleben und Geschlechterverhältnisse heute nicht mehr zusammenpassen, den Individuen aber die Verantwortung für eine gelingende Lebensgestaltung obliegt, liegt das eigentliche prekäre Potenzial von Entgrenzung. Die Folgen sind eine zunehmende Überforderung der Herstellungsleistungen von Familie einschließlich der Reproduktionskrise gegenwärtiger und zukünftiger Arbeitskräfte (Jürgens 2010). 


\section{LITERATUR}

Betzelt, S. (2008): Zur begrenzten Nachhaltigkeit flexibler Erwerbsformen - das Beispiel hoch qualifizierter Alleinselbständiger, in: Szydlik, M. (Hrsg.): Flexibilisierung - Folgen für Familie und Sozialstruktur, Wiesbaden, S. 93-112

Boushey, H. (2006): Tag Team Parenting, Washington

Brinkmann, U./Dörre, K./Röbenack, S. (2006): Prekäre Arbeit, Bonn Bundesministerium für Wirtschaft und Arbeit (BMWA) (Hrsg.) (2005): Stellenbesetzungsprozesse im Bereich „einfacher" Dienstleistungen, Berlin

Castel, R./Dörre, K. (Hrsg.) (2009): Prekarität, Abstieg, Ausgrenzung. Die soziale Frage am Beginn des 21. Jahrhunderts, Frankfurt a. M./ New York

Giddens, A. (1988): Die Konstitution der Gesellschaft, Frankfurt a. M. Gottschall, K./Voß, G. G. (2003): Entgrenzung von Arbeit und Leben, Einleitung, in: Gottschall, K./Voß, G. G. (Hrsg.): Entgrenzung von Arbeit und Leben, München, S. 11-33

Groß, H./Seifert, H./Sieglen, G. (2007): Formen und Ausmaß verstärkter Arbeitszeitflexibilisierung, in: WSI-Mitteilungen 60 (4), S. 202-208 Jurczyk, K. (2007): Ansätze zu einer emanzipatorischen Familienpolitik Der Siebte Familienbericht, in: WSI-Mitteilungen 60 (10), S. 531-537 Jurczyk, K./Schier, M./Szymenderski, P./Lange, A./Voß, G. (2009): Entgrenzte Arbeit - entgrenzte Familie, Berlin Jürgens, K. (2010): Deutschland in der Reproduktionskrise, in: Leviathan 38 (4), S. 559-587

Kremer-Sadlik, T./Paugh, A. (2007): Everyday Moments, in: Time and Society 16 (2/3), S. 287-308
Lantermann, E.-D./Döring-Seipel, E./Eierdanz, F./Gerhold, L. (2009): Selbstsorge in unsicheren Zeiten, Weinheim

Lesnard, L. (2008): Off-Scheduling within Dual-Earner Couples, in: American Journal of Sociology 114 (2), S. 447-490

Manske, A. (2010): Paradoxien des arbeitsgesellschaftlichen Wandels, www.fes-online-akademie.de

Marrs, K. (2007): Zwischen Leidenschaft und Lohnarbeit, Berlin

Rogge, B. (2009): Entwertete Zeit? Erwerbslosenalltag in Paarbeziehung und Familie, in: Heitkötter, M. (Hrsg.): Zeit für Beziehungen?, Opladen, S. 66-89

Satzer, R. (2007): Ausgeleuchtet - Vom Arbeiten und Leben in der Filmindustrie, Hannover

Schier, M. (2010): Multilokaler Alltag beruflich mobiler Eltern - (K)ein Handlungsfeld für die betriebliche Gestaltung? in: Brandt, C. (Hrsg.): Mobile Arbeit - Gute Arbeit?, Berlin, S. 101-116

Schier, M./Szymenderski, P. (2009): Von der Vorgabe zur Aufgabe, in: Jurczyk, K./Lange, A. (Hrsg.): Vaterwerden und Vatersein heute, Gütersloh, S. 250-269

Statistisches Bundesamt (2003, 2007): Bevölkerung und Erwerbstätigkeit, Wiesbaden

Statistisches Bundesamt (2009): Niedrigeinkommen und Erwerbstätigkeit, Wiesbaden

Vereinte Dienstleistungsgewerkschaft (ver.di) (2006): Strukturwandel im Einzelhandel. Branchendaten 2005, Berlin

Voss-Dahm, D. (2004): Ankerkräfte im alltäglichen Chaos, in: Mitbestimmung 50 (12), S. 30-33 